\title{
interview
}

\section{Light-driven take-off}

\section{Light can now be used to raise a wing-shaped refractive object, in a technique analogous to aerodynamic lift. Grover Swartzlander from the Rochester Institute of Technology in the USA told Nature Photonics how his team achieved optical lift using a uniform collimated beam of light.}

What motivated you to start this work?

I am generally curious about the ways in which fluid dynamics and aerodynamic phenomena are manifested in optical systems. After reading about the role of vortices in the flight of moths and bats, I decided to explore whether optical lift was possible using light, and whether it could be achieved in a stable and controlled way. However, I learned from aerodynamic engineers that a vortex field could create undesirable instabilities. In previous years I had also conducted research on optical tweezers, and was aware that a tightly focused beam, which is highly non-uniform, could impart momentum on a spherical particle to trap it in the focal region of the beam. With a little faith in the 'universality principle', which states that physical phenomena in one system tend to appear in other systems, I set out to explore how a uniform light field could produce optical lift, seeking an optical analogue of the aeroplane wing.

\section{What had been achieved previously?} Optical tweezers provide a means of moving particles perpendicularly to the direction of the incident light beam. They use a very high numerical aperture microscope objective to achieve a tightly focused beam, making them effective at controlling single, localized micrometre-scale spherical particles. In contrast, optical lift occurs in an unfocused or weakly focused beam, and thus may be used to move non-localized shaped particles in either the microscopic or macroscopic domain. To my knowledge, the optical lift force has not been previously explored.

\section{What have you achieved?}

Light scattering from clumped spheres or aspheres is well-understood for cases in which the size of the object is smaller than the wavelength of light. It is theoretically possible to obtain closed-form solutions in such cases, but solutions do not exist for complex bodies that are larger than the wavelength of light used. A ray-tracing approach seemed to be the only logical approach. Accounting for the multiple reflections and refractions for a non-spherical object, however, can be a nightmare. We incorporated the open-source Persistence of Vision ray-tracing software

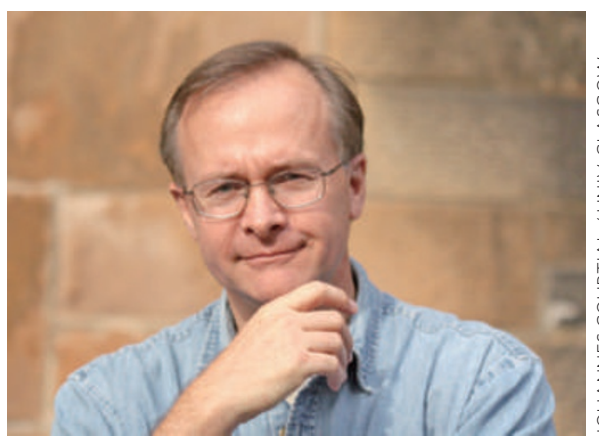

Grover Swartzlander: "Optical scientists may soon be investigating objects with alternative shapes and optical properties to achieve an adequate degree of control using optical lift."

into our computer program to calculate the forces and torques on arbitrary body shapes. We managed to construct a ray-tracing model of a uniformly illuminated, long semicylindrical transparent rod.

The shape of the object - referred to as a 'lightfoil' - is of central importance here. For the case of a semi-cylindrical rod, the model predicted that uniform illumination would cause the object to rotate towards a stable orientation and then undergo uniform lift, thereby simultaneously pushing and lifting the body in two different directions. This prediction of lift was remarkable because aerodynamic stability is difficult to achieve for an isolated wing. We then experimentally verified this stability using micrometrescale semi-cylindrical optical wings made from transparent standard photoresist. The results confirmed that when such an object is irradiated, it indeed rotates into a position of stable equilibrium and then undergoes uniform lift. Only $\sim 130 \mathrm{~mW}$ of optical power was needed to move the lightfoil at a transverse speed of a few micrometres per second. Better fabrication control over the lightfoil shape should allow lower powers of light to achieve the same transverse speed.

What is the physics behind optical lift? The optical lift is attributed to radiation pressure from the refracted and reflected rays of light on the object. Refraction and reflection both change the momentum of light, and Newton tells us that the object under illumination must therefore experience a net force and a net torque. There is interesting controversy about the momentum transfer between light and matter, owing to different interpretations of the Poynting theorem by Minkowski and Abraham. As a direct consequence of the Poynting theorem, which states that the net energy flow into a body must equal the net energy outflow unless there are sources or absorbers of energy, the predictions of our ray-tracing model were independent of whether the Abraham or Minkowski interpretation was used. However, the Minkowski model is appealing because it provides a direct understanding of optical lift in terms of a fundamental theorem of aerodynamic lift the Kutta-Joukowski theorem.

\section{What are the implications and} potential applications?

Our work represents the first step in a series of research directions. Optical scientists may soon be investigating objects with alternative shapes and optical properties to achieve an adequate degree of control using optical lift. The optical forces here are small, so do not expect to see a commuter vehicle based on optical forces. The likely scenarios are either the microscopic aqueous transport of biological tissue using light-driven micromachines, or large-scale solar sails in outer space, where small optical forces are not confounded by air friction and turbulence. The strength of the lift force can match that of the forward-scattering force, and thus relatively rapid steering and braking may be possible.

\section{How can this technique be improved?}

Fabricating microscopic lightfoils is currently a challenge for us, but promising approaches include greyscale photolithography and laser sculpting. Getting the right material properties is another challenge, as highaspect-ratio microparticles tend to curl. We therefore need to find microparticles that are stiff after they have been processed.

\section{INTERVIEW BY RACHEL WON}

Grover Swartzlander and co-workers have an Article on their optical lift technique on page 48 of this issue. 